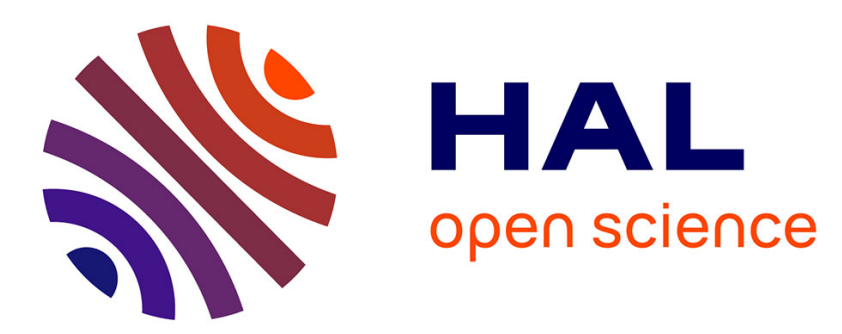

\title{
Increasing Radical Character of Large [n]cyclacenes Unveiled by Wave Function Theory
}

Stefano Battaglia, Noelia Faginas Lago, Dirk Andrae, Stefano Evangelisti, Thierry Leininger

\section{- To cite this version:}

Stefano Battaglia, Noelia Faginas Lago, Dirk Andrae, Stefano Evangelisti, Thierry Leininger. Increasing Radical Character of Large [n]cyclacenes Unveiled by Wave Function Theory. Journal of Physical Chemistry A, 2017, 121 (19), pp.3746-3756. 10.1021/acs.jpca.7b00123 . hal-01527499

\section{HAL Id: hal-01527499 \\ https://hal.science/hal-01527499}

Submitted on 18 Sep 2018

HAL is a multi-disciplinary open access archive for the deposit and dissemination of scientific research documents, whether they are published or not. The documents may come from teaching and research institutions in France or abroad, or from public or private research centers.
L'archive ouverte pluridisciplinaire HAL, est destinée au dépôt et à la diffusion de documents scientifiques de niveau recherche, publiés ou non, émanant des établissements d'enseignement et de recherche français ou étrangers, des laboratoires publics ou privés. 


\section{ACCEPted MANuscRipt}

This document is the Accepted Manuscript version of a Published Work that appeared in final form in Journal of Physical Chemistry A, copyright (c) American Chemical Society after peer review and technical editing by the publisher. To access the final edited and published work see

http://pubs .acs .org/articlesonrequest/AOR-IXb3jg3tKRE5TnwttCd9

DOI: https://doi.org/10.1021/acs.jpca.7b00123 


\section{Increasing Radical Character of Large $[n]$ cyclacenes Unveiled by Wavefunction Theory}

Stefano Battaglia, ${ }^{+\ddagger}$ Noelia Faginas-Lago, ${ }^{\ddagger}$ Dirk Andrae, ${ }^{\mathbb{I I}}$ Stefano Evangelisti, ${ }^{+}$ and Thierry Leininger*,+

+Laboratoire de Chimie et Physique Quantiques, IRSAMC, Université de Toulouse, CNRS, UPS, 118 Route de Narbonne, 31062 Toulouse Cedex, France

$\ddagger$ Dipartimento di Chimica, Biologia e Biotecnologie, Università degli Studi di Perugia, Vie Elce di Sotto 8, 06123 Perugia, Italy

IIPhysikalische und Theoretische Chemie, Institut für Chemie und Biochemie, Freie Universität

Berlin, Takustraße 3, 14195 Berlin, Germany

E-mail: Thierry.Leininger@irsamc.ups-tlse.fr

Phone: +33 (0)5 61556152 



\begin{abstract}
We have investigated the radicality and the vertical singlet-triplet energy gap of $[n]$ cyclacenes (cyclic polyacenes) as a function of the system size for $n$ even, from 6 to 22. The calculations are performed using the complete active space self-consistent field method and second-order $n$-electron valence perturbation theory. We present a systematic way for the selection of the active space in order to have a balanced description of the wavefunction as the size of the system increases. Moreover, we provide didactic insight into the failure of an approach based on a minimal active space. We find that the ground state is an open-shell singlet and its multireference character increases progressively with $n$. The singlet-triplet gap decreases as a function of the system size and approaches a finite positive value for the limit $n \rightarrow \infty$. Finally, an analysis based on the one-particle reduced density matrix suggests a polyradical character for the largest cyclacenes.
\end{abstract}

\title{
Introduction
}

Since the discovery of carbon nanotubes (CNTs) in $1991,{ }^{1}$ the field studying low-dimensional hydrocarbons has seen a tremendous increase of interest by research groups all over the world. Among the vast domain of possible structures, $[n]$ cyclacenes (rolled-up polyacenes in a ring-like shape with zigzag edges) have been challenging both experimentalists and theoreticians for the past 25 years. In fact, these molecules appeared for the first time in the literature in a Hückel study by Heilbronner ${ }^{2}$ in 1954.

The interesting and unusual properties arising from the cyclization of polyacenes have been already noticed in theoretical works applying semi-empirical methods, especially in the late $90^{\prime}$ s by Türker ${ }^{3-6}$ and Gutman, 7,8 and by André 9,10 in 2001. First-principles calculations based on density functional theory (DFT) proposed by Choi and Kim ${ }^{11}$ complemented these results by investigating the singlet-triplet energy gap (ST gap), the 
carbon-carbon bond lengths and magnetic properties as a function of the system size. The authors of that study considered $[n]$ cyclacenes as two $[2 n]$ trannulenes stacked on top of each other, such that a comparison of the properties and the aromatic character could be made with respect to their two constituting fragments. On the other hand, the relation to their open-ended "cousins", the linear polyacenes, is evident. From the semi-empirical studies, it is known that the behavior of $[n]$ cyclacenes for odd and even $n$ values is different. In particular, in the latter case the electronic structure is similar to that of polyacenes, thus sharing the same difficulties in the determination of the ground state which led to controversial findings. Indeed, this was the case also for cyclacenes, where a slightly increasing ST gap as a function of $n$ was predicted by both Choi and $\mathrm{Kim}^{11}$ and Houk et al. ${ }^{12}$ using DFT. It was only a few years later that Chen et al. ${ }^{13}$ and Sadowsky et al. ${ }^{14}$ showed by performing more accurate multireference calculations based on wavefunction theory that the increasing gap was the result of a singlet instability of the ground state computed by DFT. A recent study by Wu et al. ${ }^{15}$ explored the electronic properties of $[n]$ cyclacenes by thermally-assisted-occupation DFT (TAO-DFT) and found agreement with the works based on high-level ab-initio methods.

The challenges posed by $[n]$ cyclacenes to today's quantum chemical approaches are not the only reason for their investigation. The interest as conceptual models for understanding more complicated hydrocarbons, their promising electronic and magnetic properties similar to linear acenes and the potential for applications in host-guest chemistry justify already this theoretical work. ${ }^{11,12,16,17}$ Nonetheless, probably the most exciting characteristic of these belt-shaped molecules is their remarkable similarity to carbon nanotubes. Much effort has been invested in trying to synthesize these compounds, and the crucial obstacle has been identified to be their high reactivity due to the radical nature of their electronic ground state. ${ }^{18,19}$

Cyclacenes constitute the last minimal building unit of carbon nanotubes that have not yet been synthesized, as the basic blocks of armchair and chiral nanotubes are already 
available. Hence, one of the major interests of such structures is their potential for the controlled synthesis in a bottom-up fashion of finite-length zigzag carbon nanotubes. ${ }^{20}$ Nonetheless, a series of similar cyclic hydrocarbons have been lately proposed both theoretically and experimentally, which allowed the successful growth of finite-size CNTs. ${ }^{21-24}$ Despite the fruitful activity of the recent years, theoretical works using high-level wavefunction theory on $[n]$ cyclacenes are still far from being exhaustive. For example, $[14]$ cyclacene is the largest ring considered so far theoretically by wavefunction theory, ${ }^{13}$ while attempts to synthesize [18]cyclacene have been reported in the literature. ${ }^{25}$ The extrapolation of the singlet-triplet energy gap to the asymptotic limit as well as a characterization of the polyradical behavior with respect to the system size, although recently investigated by a DFT-based approach, ${ }^{15}$ are still missing from a wavefunction perspective.

In the present contribution we try to shed light on some of these points by complementing and extending the work carried out by other authors, ${ }^{13-15}$ treating $[n]$ cyclacenes up to $n=22$ using a multiconfigurational approach and multireference many-body perturbation theory. This allowed us to unveil the polyradical character of these systems and to provide computational evidence of the decreasing ST gap as a function of the system size. We introduce a systematic approach to select the active space (AS) for the multiconfigurational calculations in order to maintain a balanced description of the wavefuntion throughout the entire series of cyclacenes studied. Moreover, we investigate the dramatic failure resulting from a minimal active space, evidencing again the difficulties that this typology of hydrocarbons poses to electronic structure methods.

The article is organized as follows. In the next section, Computational Details, we list the theoretical methods and the important parameters chosen for this study, accompanied by a discussion of the $[n]$ cyclacenes' geometries used in the calculations. In the Results section, we divide the presentation of our findings into two parts, the first one elucidating the reasons of the failing minimal AS, and the second one presenting the results obtained with the larger AS along with the radicality analysis based on the one-particle density 
matrix. Finally, in the Conclusions section we wrap up the discussion and summarize the main achievements of this work. 


\section{Computational Details}

All the calculations were performed using the 2015.1 version of the MOLPRO program package ${ }^{26,27}$ applying the default convergence thresholds. The basis set used throughout the entire work is the atomic natural orbital (ANO) split-valence double- $\zeta$ basis set by Roos and coworkers, ${ }^{28}$ without (ANO-DZ) and with (ANO-DZP) a set of polarization functions on the carbon atoms. To ensure the quality of the results with respect to the basis set chosen, calculations on a [8]cyclacene and a [20]cyclacene have been performed using a larger triple- $\zeta$ basis set, without (ANO-TZ) and with (ANO-TZP) a set of polarization functions on the carbon atoms. As it will be discussed in more details in the Results section, the use of a larger basis did not affect the results in a significant way.

$[n]$ cyclacenes are highly symmetric molecules, hence in order to maintain the full $D_{\text {nh }}$ molecular point group, geometrical constraints have been imposed during the optimization procedure. However, the symmetry of the wavefunction is explicitly treated only by the highest abelian subgroup (i.e. $D_{2 h}$ ) due to limitations of the software employed and the labels of the states used throughout this work refer to that subgroup only. All the geometries were relaxed at restricted Hartree-Fock (RHF) level using the ANO-DZ basis set, optimizing a totally symmetric closed-shell singlet $\left({ }^{1} A_{g}\right.$ state) and manually imposing the occupation pattern of the molecular orbitals (MOs) for each value of $n$ (Table S1 in the Supplementary Information). To assess the quality of the geometries obtained at this level of theory, a series of additional optimizations has been performed on a $[8]$ cyclacene using restricted (RB3LYP) and unrestricted (UB3LYP) DFT with the three-parameter Becke exchange energy functional ${ }^{29,30}$ and the Lee-Yang-Parr correlation energy functional ${ }^{31}$ and restricted second-order Møller-Plesset perturbation theory (RMP2) using different contractions of the ANO basis set. A comprehensive table containing the geometrical parameters for all the combinations of basis set sizes and methods is available in the Supplementary Information (Table S2). The geometries obtained with the highest 
level of theory considered (RMP2/ANO-TZP and RB3LYP/ANO-TZP) differ from the geometry used in the calculations by less than $1 \%$ for all structural parameters (Table S3). In particular the largest deviations are observed for the parameters involving the hydrogen atoms. To test if the same deviation is observed on larger systems, a medium size [16]cyclacene as well as a large [20]cyclacene have been optimized at RB3LYP / ANO-DZP level of theory. In this case, apart from the $\mathrm{C}-\mathrm{H}$ bond length (with a deviation of $1.5 \%$ from the RHF / ANO-DZ geometries) all the parameters obtained are within $0.8 \%$ from the geometries used in this work (Table S4).

In this work, the complete active space self-consistent field (CASSCF) method ${ }^{32,33}$ has been employed, complemented by partially contracted second-order $n$-electron valence perturbation theory (NEVPT2) ${ }^{34-36}$ calculations where possible. In the CASSCF procedure, two states of different spin multiplicity have been optimized in the state-average fashion with equal weights, namely the lowest singlet ${ }^{1} A_{g}$ state and the lowest triplet ${ }^{3} B_{1 u}$ state (within the subgroup $D_{2 h}$ ). All orbitals have been optimized simultaneously for both states, including the $\sigma$ core ones. Two sets of active space were chosen for the CASSCF calculations; i) a minimal two electrons in two orbitals (denoted $(2,2)$ ) and ii) a dynamically sized space generated by a selection rule based on the Hückel approximation (denoted $(k, k))$. For the AS i), surprisingly wrong results came out from the calculations and an investigation of the nature of this failure has been carried out. The AS ii) consisted of a subset of the valence $\pi$ orbitals, selected in a systematic way according to a precise rule which will be described in detail in the Results section.

For systems up to $n=16$, state-specific NEVPT2 calculations have been performed using the state-average natural orbitals (NOs) obtained from the CASSCF procedure, freezing the $\sigma$ core orbitals.

In this work, the vertical singlet-triplet energy gap has been computed as simple energy differences $\left(E_{T_{1}}-E_{S_{0}}\right)$ at both the CASSCF and the NEVPT2 level of theory. As a last remark, the effects of the geometry on this energy difference appear to be minimal, as in 
the comparison of the RHF/ANO-DZ with the RB3LYP/ANO-DZP geometries virtually no change of this energy difference has been observed for [16]- and [20]cyclacene, while only a small change of $\approx 0.03 \mathrm{eV}$ was found for [8]cyclacene (Tables S5 and S6). 


\section{Results and Discussion}

The electronic structure of $[n]$ cyclacenes shares some similarities with that of linear polyacenes. At the RHF level, the highest occupied molecular orbital (HOMO) and the lowest unoccupied one (LUMO) are completely delocalized over the two zigzag edges, as depicted in Figure 1 for [10]cyclacene. Note that the orbital of Figure 1a is symmetric with respect to reflection through the horizontal plane, where as the orbital of Figure $1 \mathrm{~b}$ is antisymmetric. This symmetry property is characteristic of the HOMO-LUMO pair of all even-numbered $[n]$ cyclacenes. Thus, in the following we will denote the symmetric MO of Figure 1a as $\pi_{+}$, and the antisymmetric MO of Figure $1 \mathrm{~b}$ as $\pi_{-}$.

We inform the reader already at this point that the nature of all the valence $\pi$ orbitals remains the same irrespective of the level of theory used to calculate them: Hückel, canonical and natural orbitals always have the same shape, and we therefore will keep the same $\pi_{+}$ and $\pi_{-}$notation to refer to them.

\section{Minimal Active Space}

Table 1 shows the CASSCF results for the minimal active space of two electrons in two orbitals using the ANO-DZ basis set (this basis set is used for all the calculations with the minimal active space). The singlet-triplet energy gap is surprisingly increasing as a function of the system size $n$. Besides the three smallest cycles, we clearly see that the tendency of the ground state is to become of closed-shell character as the size parameter $n$ of the cyclacene increases. The occupation of the $\pi_{-}$orbital reaches almost two for the largest system, and consistently the weights $C_{-}$of the associated $\left(\pi_{-}\right)^{2}$ configuration show a value close to one. For $n=6,8,10$ we observe a ground state where the $\pi_{+}$orbital is more occupied than its antisymmetric counterpart.

For all cyclacenes there is a clear trend: the natural orbital occupation number (NOON) of 
$\pi_{+}$monotonically decreases towards zero, while that of $\pi_{-}$increases towards two as the system increases in size. Moreover, this trend is accompanied by a decrease of the weight $C_{+}$of the configuration $\left(\pi_{+}\right)^{2}$ and an increase of the weight $C_{-}$of the configuration $\left(\pi_{-}\right)^{2}$. These results are in contradiction with the most recent theoretical studies ${ }^{14,15}$ and our results using a larger active space, as it will be shown in the next subsection. Although $[n]$ cyclacenes are known to possess an open-shell ground state, calculations with this AS fail to correctly describe the nature of the wavefunction when the size of the system grows; instead of an increase of the multireference character, the ground state becomes increasingly closed-shell in character as $n$ increases. To investigate what drives the failure of the minimal AS, we studied the individual contributions to the correlation energy introduced by the active space part.

The CASSCF wavefunction $\left|\Psi^{C A S}\right\rangle$ can be factorized into a core determinantal part $\left|\Phi_{c}\right\rangle$ and an active space part $\left|\Psi_{a}\right\rangle$ such that it can be expressed as the tensor product $\left|\Phi_{c}\right\rangle \otimes$ $\left|\Psi_{a}\right\rangle=\left|\Phi_{c} \Psi_{a}\right\rangle=\left|\Psi^{C A S}\right\rangle$. For our particular case, the active space part of the singlet ground state is given by

$$
\left|\Psi_{a}\right\rangle=C_{+}\left|\Phi_{+}\right\rangle+C_{-}\left|\Phi_{-}\right\rangle=C_{+}\left|\psi_{+} \bar{\psi}_{+}\right\rangle+C_{-}\left|\psi_{-} \bar{\psi}_{-}\right\rangle,
$$

where $\psi_{\{+,-\}}=\alpha \pi_{\{+,-\}}$and $\bar{\psi}_{\{+,-\}}=\beta \pi_{\{+,-\}}$denote the alpha and the beta spin orbitals of the corresponding symmetric and antisymmetric $\pi_{\{+,-\}}$molecular orbitals exemplified in Figure 1, respectively. Note that although $\left|\psi_{+} \bar{\psi}_{-}\right\rangle$and $\left|\bar{\psi}_{+} \psi_{-}\right\rangle$have spin multiplicity equal to one, they do not contribute to the singlet ground state wavefunction because they do not belong to the same irrep as $\left|\Psi_{a}\right\rangle$.

The CASSCF total energy is given by

$$
\left\langle\Psi^{C A S}|\hat{\mathcal{H}}| \Psi^{C A S}\right\rangle=E_{\text {core }}+\left\langle\Psi_{a}|\hat{\mathcal{H}}| \Psi_{a}\right\rangle=E_{\text {core }}+E_{\text {corr }}
$$


where the electron correlation energy $E_{\text {corr }}$ due to the active space part of the wavefunction can be further decomposed into one- and two-electron contributions as

$$
\left\langle\Psi_{a}|\hat{\mathcal{H}}| \Psi_{a}\right\rangle=E_{\text {corr }}=E_{1}+E_{2}
$$

with $E_{1}$ and $E_{2}$ given by

$$
\begin{aligned}
& E_{1}=2 C_{+}^{2}\left(\pi_{+}|h| \pi_{+}\right)+2 C_{-}^{2}\left(\pi_{-}|h| \pi_{-}\right), \\
& E_{2}=C_{+}^{2}\left(\pi_{+} \pi_{+} \mid \pi_{+} \pi_{+}\right)+C_{-}^{2}\left(\pi_{-} \pi_{-} \mid \pi_{-} \pi_{-}\right)+2 C_{+} C_{-}\left(\pi_{-} \pi_{+} \mid \pi_{-} \pi_{+}\right) .
\end{aligned}
$$

The terms enclosed in round brackets are one- and two-electron integrals over the two orbitals of the active space.

The two-electron integrals on a delocalized basis set go to zero with respect to the system size; this means that the larger the ring gets, the more the energy is dominated by the one-electron terms. Thus, for our case, the values of $\left(\pi_{+}|h| \pi_{+}\right)$and $\left(\pi_{-}|h| \pi_{-}\right)$are crucial to determine the character of the wavefunction in the limit of large $n$. If their difference is significant, the ground state wavefunction will be predominantly described by the determinant including the orbital with the associated lowest one-electron integral (or, equivalently, the largest in absolute terms).

In Figure 2 the two-electron integrals of type $\left(\pi_{+} \pi_{+} \mid \pi_{+} \pi_{+}\right)$and $\left(\pi_{-} \pi_{+} \mid \pi_{-} \pi_{+}\right)$are plotted against the system size for $n=8,12,16, \ldots, 28$. Note that the integrals of type $\left(\pi_{-} \pi_{-} \mid \pi_{-} \pi_{-}\right)$are omitted since they are practically indistinguishable from $\left(\pi_{+} \pi_{+} \mid \pi_{+} \pi_{+}\right)$. For both types, a rapid decay obeying a power law is confirmed by our numerical results. The model function $f(n)=b n^{-c}$ falling towards zero is fitted to the integrals' values and the optimal exponents are found to be $c=-0.65$ for $\left(\pi_{+} \pi_{+} \mid \pi_{+} \pi_{+}\right)$and $c=-1.0$ for $\left(\pi_{-} \pi_{+} \mid \pi_{-} \pi_{+}\right)$. The one-electron terms depicted in Figure 3 show a negative gap at $n=8$, which becomes positive at $n=12$ and steadily increases with $n$, up to $n=28$. There is a direct relationship between the gap $\delta=\left(\pi_{+}|h| \pi_{+}\right)-\left(\pi_{-}|h| \pi_{-}\right)$and the coefficients 
$C_{+}$and $C_{-}$, as well as the occupations of the two MOs of the active space. An increasing positive gap $\delta$ corresponds to an increase of both the weight $C_{-}$and the occupation of the antisymmetric $\mathrm{MO} \pi_{-}$. Besides $n=8$, there is also a relationship between $\delta$ and the ST gap, as both increase with increasing system size. The trends of the one- and two-electron integrals with respect to the size of the cyclacenes explain why we observe a closed-shell ground state for large $n$ values.

The one-electron integrals gap $\delta$, and therefore also the singlet-triplet energy gap, depend on the shape of the molecular orbitals $\pi_{+}$and $\pi_{-}$. The orbitals optimized within the small AS and using a small double- $\zeta$ basis set might lack the flexibility to correctly relax, thus resulting in natural orbitals (NOs) which produce an artificially increasing gap $\delta$ (although the effect of the basis set size does not influence dramatically the ST gap, see Table 2). To investigate if this is the case, complete active space configuration interaction (CASCI) calculations with the minimal AS of two electrons in two orbitals have been performed using $\operatorname{CASSCF}(8,8)$ NOs and CASSCF $(16,16)$ NOs on [8]cyclacene and [20]cyclacene, respectively. This choice of natural orbitals is motivated by the systematic selection approach discussed in the next subsection and by the corresponding CASSCF calculations which produced results in line with the existing literature. Therefore, we consider these two sets of orbitals as qualitatively correct for the two particular $[n]$ cyclacenes analyzed. The results listed in Table 3 show for both systems an increase of the singlet-triplet gap using the correctly relaxed orbitals. In particular, for $n=8$ the difference between the two gaps is $0.006 \mathrm{eV}$, whereas in the larger [20]cyclacene the gap goes from $0.50 \mathrm{eV}$ to $0.61 \mathrm{eV}$. Hence, it appears that the $\operatorname{CASSCF}(2,2)$ NOs have the effect to underestimate the ST gap, suggesting that the minimal active space is actually limiting the increase of the gap with respect to the system size, rather than artificially creating it by the lack of flexibility. Corroborating this picture, the one-electron integrals computed using $\operatorname{CASSCF}(8,8)$ and $\operatorname{CASSCF}(16,16) \operatorname{NOs}$ shift the gap $\delta$ by $+0.03 \mathrm{eV}$ and $+0.12 \mathrm{eV}$ for [8]cyclacene and [20]cyclacene, respectively (see Table 4). The two-electron integrals appear to be unaffected by the type of orbitals, as 
their values remain virtually unchanged (Table 4).

Rationalizing this analysis of the correlation energy and the individual integrals contributions, we argue that the failure of the minimal AS is completely AS-driven and not caused by insufficiently relaxed orbitals. The AS with two electrons in two orbitals is simply too small to capture the essential physics of the system.

\section{Dynamical Active Space}

In order to obtain the correct qualitative description of the wavefunction it is required to go beyond the minimal active space. Since the systems under investigation are of increasing size, it is important to keep a balanced description of the wavefunction throughout the series of cyclacenes, such that the results obtained are comparable with each other. In particular, for AS-based methodologies one should adopt a systematic procedure to increase the number of explicitly correlated orbitals as the system grows.

A common approach applied to linear polyacenes in recent years is to include all the valence $\pi$ orbitals in the AS. $16,37,38$ However, it was not a viable option in our case since the resulting active spaces were computationally too demanding for the CASSCF method. Therefore, we have devised an alternative selection scheme based on Hückel theory. As depicted in Figure 4, one can model $[n]$ cyclacenes as $n$ units containing four carbon atoms each. The resulting Hückel Hamiltonian is given by the following block matrix

$$
\mathbf{H}_{n}=\left[\begin{array}{cccccc}
\mathbf{H}_{0} & \mathbf{H}_{1} & \mathbf{0} & \ldots & \mathbf{0} & \mathbf{H}_{-1} \\
\mathbf{H}_{-1} & \mathbf{H}_{0} & \mathbf{H}_{1} & \ldots & \mathbf{0} & \mathbf{0} \\
\ldots & \ldots & \ldots & \ldots & \ldots & \ldots \\
\mathbf{H}_{1} & \mathbf{0} & \mathbf{0} & \ldots & \mathbf{H}_{-1} & \mathbf{H}_{0}
\end{array}\right]
$$


where each entry is itself a matrix of size $4 \times 4$, as the elementary unit cell is composed by 4 atoms. The blocks $\mathbf{H}_{0}$ on the diagonal are given by

$$
\mathbf{H}_{0}=\left[\begin{array}{cccc}
\alpha & -\beta & 0 & 0 \\
-\beta & \alpha & -\beta & 0 \\
0 & -\beta & \alpha & -\beta \\
0 & 0 & -\beta & \alpha
\end{array}\right]
$$

and describe the interactions within the elementary units, while the off-diagonal blocks $\mathbf{H}_{1}$ and $\mathbf{H}_{-1}$ are given by

$$
\mathbf{H}_{1}=\left[\begin{array}{cccc}
0 & -\beta & 0 & 0 \\
0 & 0 & 0 & 0 \\
0 & 0 & 0 & 0 \\
0 & 0 & -\beta & 0
\end{array}\right] \quad \text { and } \mathbf{H}_{-1}=\left[\begin{array}{cccc}
0 & 0 & 0 & 0 \\
-\beta & 0 & 0 & 0 \\
0 & 0 & 0 & -\beta \\
0 & 0 & 0 & 0
\end{array}\right]=\left(\mathbf{H}_{1}\right)^{T}
$$

and describe the interactions across the units. Upon diagonalization of $\mathbf{H}_{n}$ through a unitary transformation, analytical expressions for its eigenvalues, or otherwise said for the orbital energies, are obtained. Moreover, these expressions are defined in the reciprocal space; as such they are periodic with period $2 \pi$. Since there are four basis functions in each unit cell, four energy bands appear in reciprocal space given by the following analytical expressions

$$
\begin{aligned}
& \varepsilon_{1}(k)=\alpha-\beta \cdot \frac{+1+\sqrt{9+8 \cos (k \theta)}}{2}, \\
& \varepsilon_{2}(k)=\alpha-\beta \cdot \frac{-1+\sqrt{9+8 \cos (k \theta)}}{2}, \\
& \varepsilon_{3}(k)=\alpha-\beta \cdot \frac{+1-\sqrt{9+8 \cos (k \theta)}}{2}, \\
& \varepsilon_{4}(k)=\alpha-\beta \cdot \frac{-1-\sqrt{9+8 \cos (k \theta)}}{2},
\end{aligned}
$$


where $\alpha$ and $\beta$ are the usual Coulomb and resonance integrals of Hückel theory, respectively, $\theta$ is equal to $2 \pi / n$ and $k$ can take integer values from 0 to $n-1$. By setting arbitrarily $\alpha=0$ and $\beta=1$, it is possible to plot the energy bands, as exemplified in Figure 5 for a [6]cyclacene and a [16]cyclacene. Note that each circle corresponds to a Hückel eigenvalue, i.e. to the orbital energy. Moreover, note that by going from a smaller system to a larger one, the size of the valence $\pi$ space increases, and consequently more molecular orbitals appear in the same interval of $2 \pi$. When $n$ is even, four molecular orbitals always appear at $k \theta=\pi$, whose shape is independent of $n$ and their energies are different apart for the HOMO-LUMO pair, which is energetically degenerate within this simple model (note that in Figure 5 there are actually two circles at the Fermi level for $k \theta=\pi$, one from $\varepsilon_{2}$ and one from $\left.\varepsilon_{3}\right)$.

The orbitals at $k \theta=\pi$ are of particular interest for our scheme. First, we point out that the HOMO and the LUMO associated to $\varepsilon_{2}(n / 2)=\varepsilon_{3}(n / 2)=\alpha$ correspond qualitatively to the $\pi_{+}$and $\pi_{-}$orbitals discussed in the previous section, and have the same characteristic shape as depicted in Figure 1. The other two orbitals at $k \theta=\pi$, i.e. associated to $\varepsilon_{1}(n / 2)=\alpha-\beta$ and $\varepsilon_{4}(n / 2)=\alpha+\beta$ (to which we shall refer to as $\pi_{+}^{\prime}$ and $\pi_{-}^{\prime}$ from now on), also have a characteristic shape as depicted in Figures 6a and 6b. Note that the difference between the orbitals $\pi_{\{+,-\}}$and $\pi_{\{+,-\}}^{\prime}$ is the position of the $p$-like atomic orbitals on the carbon atoms, in the latter case they are non-zero only on the central atoms, while in the former only on the external ones.

By drawing two horizontal lines passing through $\pi_{+}^{\prime}$ and $\pi_{-}^{\prime}$ in Figure 5, a delimited energetic zone (depicted in gray in Figure 7) appears containing a finite number of orbitals for finite values of $n$. Our approach consists in selecting for the active space all molecular orbitals lying in this window, including the $\pi_{+}^{\prime}$ and $\pi_{-}^{\prime}$ orbitals defining the boundaries. Since more orbitals appear along the bands when the size of the system is increased, automatically the energy window (and therefore the active space) will also contain more orbitals. 
It is important to take into account that by changing the level of theory, the ordering of the orbitals is likely to be affected. However, it turns out that their nature remains unaffected regardless of the method employed, so that $\pi_{+}^{\prime}$ and $\pi_{-}^{\prime}$ are always easily identifiable.

In this work, the selection of the dynamical active spaces was made by applying the described method on canonical Hartree-Fock orbitals. This choice generated active spaces enlarging by four orbitals and four electrons for each increase of six hexagonal units in the $[n]$ cyclacenes, i.e.

$$
\begin{gathered}
n \rightarrow n+6 \\
(k, k) \rightarrow(k+4, k+4) .
\end{gathered}
$$

The resulting AS sizes for the systems considered in this work are summarized in Table 5.

The CASSCF vertical singlet-triplet energy gap calculated using both the ANO-DZ and the ANO-DZP basis sets, along with the NOONs of $\pi_{+}$and $\pi_{-}$and the configuration weights $C_{+}$and $C_{-}$of the ANO-DZP results are shown in Table 6. We predict a monotonically decreasing ST gap with system size for both basis sets. The natural orbital occupation number of $\pi_{+}$is always greater than that of $\pi_{-}$, contrary to the $\operatorname{CASSCF}(2,2)$ case and this result is accompanied by the weight of the configuration doubly occupying $\pi_{+}$, which is always greater than that doubly occupying the antisymmetric orbital.

Interestingly, the increase of size of the cyclacene has a greater impact on the weight of the symmetric configuration than that of the antisymmetric one. Indeed, from $n=6$ to $n=22$ the absolute value of the coefficient $C_{+}$goes from 0.85 down to 0.28 , while $C_{-}$only goes from 0.36 to 0.24 . In addition, both $C_{+}$and $C_{-}$decrease in magnitude as $n$ increases; with increasing AS along this homologous sequence, the two "reference configurations", $\left(\pi_{+}\right)^{2}$ and $\left(\pi_{-}\right)^{2}$, become less and less important.

For small $[n]$ cyclacenes, the decrease of the gap does not follow the same decaying be- 
havior observed for the larger ones; in particular for $n=6$ to $n=10$ the decay is clearly different from the one seen for $n>10$, as shown in Figure 8. The best fit to the energy gap is given by the exponentially decaying function $f(n)=a+b e^{-c n}$, excluding the first two points ( $n=6$ and $n=8)$ in the parameters estimation. Of special interest is the limit of the singlet-triplet energy gap as $n$ approaches infinity, predicting a value between 0.14 and $0.16 \mathrm{eV}$ for the infinite-size system.

For systems with $n$ up to 16 , dynamical electron correlation has been introduced by performing NEVPT2 calculations, in order to study its effect on the singlet-triplet energy gap. Since we are working with a restricted AS on relatively large systems, the choice of method to introduce the dynamical correlation has to be taken carefully. The well-known CASPT2 method ${ }^{39,40}$ suffers from the so-called intruder states problem if insufficiently large active spaces are considered in the underlying CASSCF calculation. This problem can be removed by the introduction of an energy shift ${ }^{41,42}$ or through the IPEA shift. ${ }^{43}$ In both cases though, the choice of the parameters is arbitrary up to a certain degree and a series of tests has to be conducted in order to assess the quality of the results. On the contrary, the NEVPT2 approach has been devised such that the problematic denominator does not appear in the expression of the second order correction to the energy. This effectively removes the problem of intruder states by design and thus it overcomes the problem given by excitations to quasi-degenerate orbitals. Table 7 lists the results of the NEVPT2 calculations. First of all the inclusion of polarization functions on the carbon atoms does not seem to have an effect on the qualitative behavior of the gap, as it was observed for the CASSCF results too. The gap slightly increases for the first three cycles and only from $n=10$ to $n=12$ it begins to go down; again in agreement with the calculated gap with the CASSCF method. For all cyclacenes the effect of dynamical correlation is to reduce the ST gap. The ST gap for $n=6,8,10$ seems to be qualitatively different than for the rest of the series with an increase as high as $0.08 \mathrm{eV}$, this behavior will be discussed later on, after the comparison of our results with those present in the literature. 
Previous theoretical studies on $[n]$ cyclacenes using wavefunction theory did not investigate the asymptotic limit of the singlet-triplet gap, this was only proposed recently by $\mathrm{Wu}$ et al. ${ }^{15}$ in a study based on DFT. In the following we will compare our results with those obtained by other authors using both wavefunction theory and DFT-based approaches. Our prediction for the ST gap is in contrast with the results of Houk et al. ${ }^{12}$, who obtained a nearly vanishing one, slightly increasing with system size. In this case the singlet state was calculated using restricted DFT and the B3LYP hybrid functional, while the triplet using unrestricted time-dependent DFT. This choice clearly overestimates the energy of the singlet state, which we here argue is an open-shell singlet, and requires a multiconfigurational approach with a sufficiently large AS in order to be correctly described. Moreover the comparison of energies calculated using restricted and unrestricted methodologies might not be balanced. In this particular case, the triplet state would be favored by the additional flexibility provided by the unrestricted approach, while the restricted singlet would result energetically too high since it is not properly described by the method. In another study, Chen et al. ${ }^{13}$ applied both unrestricted DFT and the CASSCF method combined with second-order multireference perturbation theory (MRMP2). Even if in this case unrestricted DFT was used for the singlet state too, the gap still showed the opposite behavior, increasing as a function of $n$. On the other hand their CASSCF result for [6]cyclacene with eight electrons in eight orbitals was $0.65 \mathrm{eV}$ using the $6-31 \mathrm{G}^{*}$ basis set. This result is in very good agreement with our predicted gap of $0.65 \mathrm{eV}$ using the same AS size and the ANO-DZ basis set. Inclusion of polarization functions lifts the gap to $0.75 \mathrm{eV}$, thus resulting $0.1 \mathrm{eV}$ higher. The MRMP2 gap of $0.32 \mathrm{eV}$ is also in good agreement with our results; it is $0.02 \mathrm{eV}$ smaller than the NEVPT2 one using the ANO-DZ basis set and $0.04 \mathrm{eV}$ lower compared to the ANO-DZP one.

The work of Sadowsky et al. ${ }^{14}$ provides to the best of our knowledge the most recent results on $[n]$ cyclacenes using wavefunction theory. Both unrestricted DFT and CASSCF 
with CASPT2 were applied to the series of $[n]$ cyclacenes, for $n$ going from 6 to 12 . Using the B3LYP functional, an erroneous positive and increasing ST gap was predicted, while by employing the M06-L functional, they found an increasing gap for $n=6,8,10$ which dropped down for $n=12$, yielding the smallest value in the series. The active space for the CASSCF calculations was of 8 electrons in $10 \mathrm{MOs}$, but unfortunately no numerical values were provided neither for the energy gap nor for the natural orbital occupation numbers. The CASPT2 values listed in the last column of Table 7 show an oscillating behavior as $n$ increases. This behavior is not observed in the case of NEVPT2, where for the first three cyclacenes the ST gap increases from $0.36 \mathrm{eV}$ to $0.44 \mathrm{eV}$ for the ANO-DZP basis set. In particular it was argued that the increase from a gap of $0.49 \mathrm{eV}$ for $n=10$ to a gap of 0.60 $\mathrm{eV}$ for $n=12$ was probably due to the limited size of the active space. This increase was indeed not observed in the case of NEVPT2, probably thanks to the enlargement of the AS from $(8,8)$ to $(12,12)$. Given the different choices of methods, basis sets and active spaces, a quantitative comparison between CASPT2 and NEVPT2 values does not provide any additional information. Nevertheless, the fact that in the two cases different qualitative results have been obtained, is a proof of the difficulty that this kind of systems still poses to quantum chemical methodologies.

At last, the most recent study of $[n]$ cyclacenes has been carried out by Wu et al. ${ }^{15}$ by performing thermally-assisted-occupation DFT (TAO-DFT) ${ }^{44}$ calculations on systems with $n$ values ranging from 4 to 100 . The behavior observed for the singlet-triplet energy gap is qualitatively the same, with a monotonically decreasing gap as system size increases. No extrapolation to the infinite-size system has been done, but with a system as large as [100]cyclacene the gap observed of $0.02 \mathrm{eV}(0.49 \mathrm{kcal} / \mathrm{mol})$ is a good estimate for such a limit. This value is below our prediction for $n \rightarrow \infty$, amounting to $\approx 0.15 \mathrm{eV}$. This discrepancy, although remarkable, should not surprise the reader, when considering the different methodologies applied and the different basis sets as well as geometries employed for the calculations. It is in fact important to remember that in this contribution, 
the vertical singlet-triplet energy gap has been investigated, in contrast to the work by Wu et al. ${ }^{15}$ where the triplet state has been relaxed to yield the adiabatic energy gap. TAO-DFT calculations show an increase of the ST gap from [6]cyclacene to [8]cyclacene as it was the case for our NEVPT2 results and the CASPT2 ones obtained by Sadowsky et al. ${ }^{14}$. The ST gap decreases then from $n=8$ to $n=10$ for both TAO-DFT and CASPT2, but not for NEVPT2 where it keeps increasing. The different behavior of NEVPT2 suggests that perhaps the AS size predicted by our selection method is not large enough in this particular case. Indeed, calculations with a $(12,12)$ AS (instead of the predicted $(8,8)$ AS) show a lowering of the ST gap at CASSCF/ANO-DZP level from $0.62 \mathrm{eV}$ to $0.59 \mathrm{eV}$. Moreover, the gap computed using NEVPT2 on this larger active space also provides a lower estimate by going from $0.44 \mathrm{eV}$ to $0.38 \mathrm{eV}$. This last value falls below the gap of [8]cyclacene and thus agrees with the previous works by Sadowsky et al. ${ }^{14}$ and Wu et al. ${ }^{15}$, making the ST gap obtained with NEVPT2 decrease monotonically starting at $n=8$.

It is known from studies on linear polyacenes as well as on graphene nanoribbons that the inclusion of all valence $\pi$ orbitals in the active space is important in order to account properly for the large static correlation present in these systems. ${ }^{16,37}$ This can be expected to be true also for $[n]$ cyclacenes, although it was never thoroughly tested. Using our active space selection scheme we were able to consider only a fraction of the $\pi$ valence space, which nevertheless proved sufficient to produce qualitatively correct results (in all cases but $n=10$ ). As an illustrative example, the enlargement of the active space of [12]cyclacene with respect to that of $[10]$ cyclacene was sufficient to predict the correct decaying behavior of the ST gap at NEVPT2 level of theory, in contrast to the result obtained by Sadowsky et al. ${ }^{14}$ using the CASPT2 method.

It is important however to study the influence of the AS on the $\Delta E_{T_{1}-S_{0}}$ gap. To this end, two larger active spaces have been tested for [10]cyclacene and one larger AS for [16]cyclacene. From Table 8 it is clear that for the small cyclacene the influence is significant, 
with the gap decreasing by $0.03-0.04 \mathrm{eV}$ at CASSCF level and by $0.06 \mathrm{eV}$ at NEVPT2 level as seen previously in the discussion of the NEVPT2 gap. This however is not true for the large cyclacene, where the gap remains unchanged upon enlargement to the $(16,16)$ AS. Thus, it appears that our selection method works best for the larger cyclacenes, while for the smaller ones, extra care has to be taken in order to ensure the quality of the results. As it was mentioned in the Computational Details section, the effect of the basis set size was tested too. In particular the gaps for both a small $(n=8)$ and a large $(n=20)[n]$ cyclacene were calculated using the ANO triple- $\zeta$ basis set without (ANO-TZ) and with (ANO-TZP) polarization functions on the carbons. The results listed in Table 9 show that the further extension of the basis set does not affect the ST gap. The differences between the results obtained with bases with and without polarization functions persist at the triple- $\zeta$ level too.

To establish the polyradical character of the (open-shell) singlet ground state, the natural orbital occupation numbers of the CASSCF wavefunction are plotted in Figure 9. For a better readability, the orbitals displayed are all unique empty and doubly occupied valence $\pi$ orbitals (i.e. only one orbital for each degenerate pair). The occupation of the active orbitals tends towards one as more hexagonal units are added to the cyclacene. In particular the occupation number of the highest occupied (HONO) and the lowest unoccupied (LUNO) natural orbital as well as the HONO-1 and the LUNO-1 appears to change rather quickly, while this behavior is slower for the other orbitals of the AS. This effect could possibly arise from the limited size of the active space, its sudden change every time $n$ increases by 6 (noticeable in Figure 9) or by the fact that geometries optimized for the closed-shell singlet have been used. The occupation numbers approaching to one have to be understood in this case as an increase in the radical character of the molecule; pairs of electrons doubly occupying single orbitals decouple and localize on either end of the cyclacenes (this can be easily seen by a unitary orbital transformation) singly occupying one orbital each. As a comparison, the active orbital occupation numbers (AOONs) 
resulting from TAO-DFT (note that these occupation numbers are an approximation of NOONs) behave differently; although the tendency is an increasing number of orbitals with fractional occupation tending to one as the system size increases, the way in which this happens is not the same as in the CASSCF case. Depending on the orbitals considered, the occupations oscillate getting close to one for later increase or decrease again, or they remain approximately constant for a certain range of $n$ values before changing significantly, or lastly, certain pairs of orbitals have approximately the same occupation number in limited intervals of $n$. However, it is important to note that for both methods no crossing of the HONO and LUNO ocuppation numbers is observed. An analysis of the AOONs is beyond the scope of this article, but since a similar study on linear polyacenes has been performed employing the same method, ${ }^{44,45}$ some remarks can be done considering the larger information available in the literature. A "simple" change of NOONs towards one has been observed in the case of linear polyacenes for calculations on systems up to [12] acene using the density matrix renormalization group (DMRG) and the variational two-particle reduced density matrix method (v2RDM) with the minimal basis STO-3G. ${ }^{16,46}$ A different behavior, in which a crossing of the HONO and LUNO occupation numbers appears at $n=10$, has been observed by calculations using the same v2RDM method but with a larger basis set ${ }^{47}$ and by TAO-DFT. ${ }^{44,45}$ In the second v2RDM study it has been argued that the crossing appears only when a larger (and thus more reliable) basis set is used, hence supporting the TAO-DFT results. In the case of $[n]$ cyclacenes no crossing is observed for both AOONs and NOONs, nevertheless qualitative differences still exist. In this work, a reliable ANO-DZP basis set is used (with benchmarks on selected systems with an ANO-TZP basis) and thus the differences probably do not arise from the basis set chosen; more likely the limited size of the AS or the intrinsic difference between AOONs and NOONs are the cause of this discrepancy.

To further assess the radicality of cyclacenes, we have calculated an effective number 
of unpaired electrons using an expression introduced by Head-Gordon ${ }^{48}$ based on the one-particle reduced density matrix. This measure yields an estimate of the total number of unpaired electrons present in the system and thus characterizes its radicality. This index is given by

$$
R=\sum_{i=1}^{n_{\text {orb }}} \min \left(n_{i}, 2-n_{i}\right)
$$

where $n_{i}$ is the natural orbital occupation number (NOON) of orbital $i$. It is easy to see that empty and doubly occupied orbitals do not contribute to $R$, and the sum can therefore be restricted to the orbitals included in the AS. The computed values are reported in Figure 10 where the radical index $R$ highlights clearly the "jumps" to larger active spaces that were less obvious in Figure 9. Note the clear trend of $R$ : for $n=6,8,10$ the radical index is between 1 and 2, for $n=12,14,16$ between 3 and 4, and finally for $n=18,20,22$ between 5 and 6 . The basis set dependence appears to be minimal, as the two curves are almost superimposed. For the first three cyclacenes there is a slightly slower increase of the index than for the larger rings, which is in agreement with slower decrease of the singlet-triplet energy gap, suggesting a close relationship. We point out that the radical index provides a lower bound for the radicality of the system, in particular the larger the AS, the more unpaired electrons are predicted by $R$.

The analysis based on the one-particle density matrix provides strong evidence of polyradical character for systems with $n>10$ with an open-shell singlet ground state. Similar conclusions have been drawn for linear polyacenes, $16,17,37,44-47,49$ although it has been argued that for systems up to heptacene, the ground state can be regarded as closed-shell and therefore single reference methods are able to predict the electronic properties within chemical accuracy. ${ }^{50}$ Nevertheless, the increase in radicality with system size is undeniable and therefore an appropriate method able to account for static correlation effects has to be used. 


\section{Conclusions}

We have studied the vertical singlet-triplet energy gap and the polyradical character of $[n]$ cyclacenes for increasing $n$ using high-level wavefunction methods. The singlet-triplet gap was found to exponentially decrease with system size $n$, and to approach a finite value in the order of a tenth of $\mathrm{eV}$ in the limit $n \rightarrow \infty$. A criterion to dynamically select the active space as a function of $n$ has allowed a balanced treatment of the static electron correlation throughout the series. Our results are compared to previous results from the literature. They extend these results and confirm the open-shell singlet nature of the electronic ground state. The increasing polyradical character has been studied by means of an analysis on natural orbital occupation numbers and of a measure of unpaired electrons. Overall qualitative agreement to the closely related linear polyacenes has been found. Moreover, calculations using a minimal active space of two electrons in two orbitals have been performed and insight into the failure of this AS has been provided through a scrupulous analysis of the integral contributions to the correlation energy. With the wrong choice of AS, the ground state of the system increasingly tends to be of closed-shell rather than open-shell character as $n$ increases. Besides the results provided, this study didactically shows the importance of the choice of the active space for this kind of systems and proposes a criterion for its systematic selection.

To conclude, although $[n]$ cyclacenes have not been synthesized yet, they remain a fertile ground for theoreticians and experimentalists to increase the basic understanding of hydrocarbon systems. 


\section{Supporting Information Available}

Molecular orbitals occupation patterns imposed for the geometry optimization, an exhaustive comparison of the [8]cyclacene geometry optimized at different levels of theory as well as different basis set sizes are available. The geometries of [16]- and [20]cyclacenes optimized at a higher level of theory than that used throughout the work as well as the effect

of the different geometries on the ST gap is tabulated. The geometries of all $[n]$ cyclacenes used in the calculations are provided. 


\section{Acknowledgement}

This work has received funding from the European Union's Horizon 2020 research and innovation programme under the Marie Skłodowska-Curie grant agreement nº642294. The calculations of this work have been performed using the resources of the HPC center CALMIP under the grant 2016-p1048. D. A. acknowledges support for a visiting grant from the Programme Investissements d'Avenir ANR-11- IDEX-0002-02, reference ANR10-LABX-0037-NEXT. N. F.-L. acknowledges financial support from Fondazione Cassa di Risparmio di Perugia (P 2014/1255, ACT 2014/6167). Finally, S. B. acknowledges the colleague Jean-Paul Malrieu for insightful discussions. 
Table 1: ST gaps, Natural Orbital Occupation Numbers of $\pi_{+}$and $\pi_{-}$for the Singlet State $S_{0}$ and Weights of the Two Configurations of the CASSCF $(2,2)$ Wavefunction.

\begin{tabular}{c||cc|cc|c}
\multicolumn{1}{c||}{} & \multicolumn{2}{c}{$\Delta E_{T_{1}-S_{0}}$} & \multicolumn{2}{c}{ NOON $S_{0}$} & \multicolumn{2}{c}{ conf. weight } \\
$n$ & $(\mathrm{eV})$ & $\pi_{+}$ & $\pi_{-}$ & $C_{+}$ & $C_{-}$ \\
\hline 6 & 0.07 & 1.32 & 0.68 & -0.81 & 0.58 \\
8 & 0.02 & 1.22 & 0.78 & -0.78 & 0.62 \\
10 & -0.003 & 1.01 & 0.99 & -0.71 & 0.70 \\
12 & 0.03 & 0.71 & 1.29 & -0.60 & 0.80 \\
14 & 0.12 & 0.43 & 1.57 & -0.46 & 0.89 \\
16 & 0.24 & 0.25 & 1.75 & -0.35 & 0.94 \\
18 & 0.37 & 0.15 & 1.85 & -0.27 & 0.96 \\
20 & 0.50 & 0.10 & 1.90 & -0.22 & 0.98 \\
22 & 0.62 & 0.07 & 1.93 & -0.18 & 0.98 \\
24 & 0.73 & 0.05 & 1.95 & -0.15 & 0.99 \\
28 & 0.91 & 0.03 & 1.97 & -0.11 & 0.99
\end{tabular}

Table 2: Effect of the Basis Set on the CASSCF(2,2) ST Gap for [8]cyclacene and for [20]cyclacene.

\begin{tabular}{c||cccc}
\multicolumn{1}{l||}{} & \multicolumn{4}{c}{$\Delta E_{T_{1}-S_{0}}(\mathrm{eV})$} \\
$n$ & ANO-DZ & ANO-DZP & ANO-TZ & ANO-TZP \\
\hline 8 & 0.02 & 0.09 & 0.02 & 0.09 \\
20 & 0.50 & 0.36 & 0.51 & 0.36
\end{tabular}


Table 3: $\mathrm{NO}(2,2)$ Refers to the CASCI $(2,2)$ ST Gap Computed Using NOs from CASSCF(2,2) Calculations, while $\mathrm{NO}(k, k)$ Computed Using NOs from CASSCF $(8,8)$ and $\operatorname{CASSCF}(16,16)$ for $[8]$ cyclacene and $[20]$ cyclacene, Respectively.

\begin{tabular}{c||r|ll|ll|l}
\multicolumn{1}{l||}{} & \multicolumn{2}{c}{$\Delta E_{T_{1}-S_{0}}(\mathrm{eV})$} & \multicolumn{2}{c}{ NOON $S_{0}$} & \multicolumn{2}{c}{ conf. weight } \\
$n$ & $\mathrm{NO}(2,2)$ & $\mathrm{NO}(k, k)$ & $\pi_{+}$ & $\pi_{-}$ & $C_{+}$ & $C_{-}$ \\
\hline 8 & 0.021 & 0.015 & 1.20 & 0.80 & 0.77 & -0.63 \\
20 & 0.50 & 0.61 & 0.08 & 1.92 & 0.20 & -0.98
\end{tabular}

Table 4: One-electron Integral Difference $\delta$ and Two-electron Integral Terms for [8]cyclacene and [20]cyclacene. Integrals Computed over CASSCF Natural Orbitals from Calculations With Two Different Active Spaces. All the Values Are Given in eV.

\begin{tabular}{|c|c|c|c|c|}
\hline \multirow[b]{2}{*}{ integral term } & \multicolumn{2}{|c|}{ [8]cyclacene } & \multicolumn{2}{|c|}{ [20]cyclacene } \\
\hline & $\operatorname{CAS}(2,2)$ & $\operatorname{CAS}(8,8)$ & $\operatorname{CAS}(2,2)$ & CAS $(16,16)$ \\
\hline$\delta$ & -0.17 & -0.14 & 0.81 & 0.93 \\
\hline$\left(\pi_{+} \pi_{+} \mid \pi_{+} \pi_{+}\right)$ & 3.8436 & 3.8627 & 2.1327 & 2.1447 \\
\hline$\left(\pi_{-} \pi_{+} \mid \pi_{-} \pi_{+}\right)$ & 0.9416 & 0.9556 & 0.3765 & 0.3847 \\
\hline
\end{tabular}

Table 5: Active Space Sizes Resulting from the Hückel-based Selection Scheme.

\begin{tabular}{c||c}
$n$ & $(k, k)$ \\
\hline $6,8,10$ & $(8,8)$ \\
$12,14,16$ & $(12,12)$ \\
$18,20,22$ & $(16,16)$
\end{tabular}

Table 6: CASSCF $(k, k)$ ST Gap, NOONs of $\pi_{+}$and $\pi_{-}$for the Singlet State ${ }^{1} A_{g}$ and Weights of the Two Dominant Configurations.

\begin{tabular}{c||ccc|cc|c}
\multicolumn{1}{l||}{} & \multicolumn{2}{c}{$\Delta E_{T_{1}-S_{0}}(\mathrm{eV})$} & \multicolumn{2}{c}{ NOON $S_{0}$} & \multicolumn{2}{c}{ conf. weight } \\
$n$ & ANO-DZ & ANO-DZP & $\pi_{+}$ & $\pi_{-}$ & \multicolumn{1}{c}{$C_{+}$} & $C_{-}$ \\
\hline 6 & 0.65 & 0.76 & 1.61 & 0.39 & 0.85 & -0.36 \\
8 & 0.59 & 0.72 & 1.62 & 0.38 & 0.84 & -0.33 \\
10 & 0.50 & 0.62 & 1.58 & 0.42 & 0.82 & -0.33 \\
12 & 0.39 & 0.44 & 1.39 & 0.61 & 0.67 & -0.34 \\
14 & 0.30 & 0.32 & 1.31 & 0.69 & 0.60 & -0.34 \\
16 & 0.24 & 0.24 & 1.25 & 0.75 & 0.53 & -0.33 \\
18 & 0.23 & 0.20 & 1.18 & 0.82 & 0.36 & -0.29 \\
20 & 0.20 & 0.18 & 1.18 & 0.83 & 0.32 & -0.26 \\
22 & 0.18 & 0.16 & 1.17 & 0.83 & 0.28 & -0.24
\end{tabular}


Table 7: Predicted ST Gap Using the NEVPT2 Method and CASPT2 Values Taken from the Literature.

\begin{tabular}{c||cc|c}
\multicolumn{1}{c||}{} & \multicolumn{3}{c}{$\Delta E_{T_{1}-S_{0}}(\mathrm{eV})$} \\
$n$ & ANO-DZ & ANO-DZP & CASPT2 $^{a}$ \\
\hline 6 & 0.34 & 0.36 & 0.52 \\
8 & 0.36 & 0.41 & 0.63 \\
10 & 0.37 & 0.44 & 0.49 \\
12 & 0.29 & 0.33 & 0.60 \\
14 & 0.24 & 0.25 & - \\
16 & 0.19 & 0.18 & - \\
\multicolumn{1}{l}{$\mid$} & \multicolumn{3}{c}{ Results reported from Ref. 14, 6-31G(d) basis }
\end{tabular}

Table 8: Effect of the Active Space Size on the CASSCF ST Gap for [10]cyclacene and [16]cyclacene Using the ANO-DZP Basis. All Values Are Given in eV.

\begin{tabular}{c||ccc}
$n$ & $(8,8)$ & $(12,12)$ & $(16,16)$ \\
\hline 10 & 0.62 & 0.59 & 0.55 \\
16 & - & 0.24 & 0.24
\end{tabular}

Table 9: Effect of the Basis Set on the CASSCF $(k, k)$ ST Gap for [8]cyclacene and for [20]cyclacene.

\begin{tabular}{c|c||cccc} 
& \multicolumn{1}{|c||}{} & \multicolumn{5}{c}{$\Delta E_{T_{1}-S_{0}}(\mathrm{eV})$} \\
$n$ & $(k, k)$ & ANO-DZ & ANO-DZP & ANO-TZ & ANO-TZP \\
\hline 8 & $(8,8)$ & 0.59 & 0.72 & 0.60 & 0.72 \\
20 & $(16,16)$ & 0.20 & 0.18 & 0.20 & 0.18
\end{tabular}




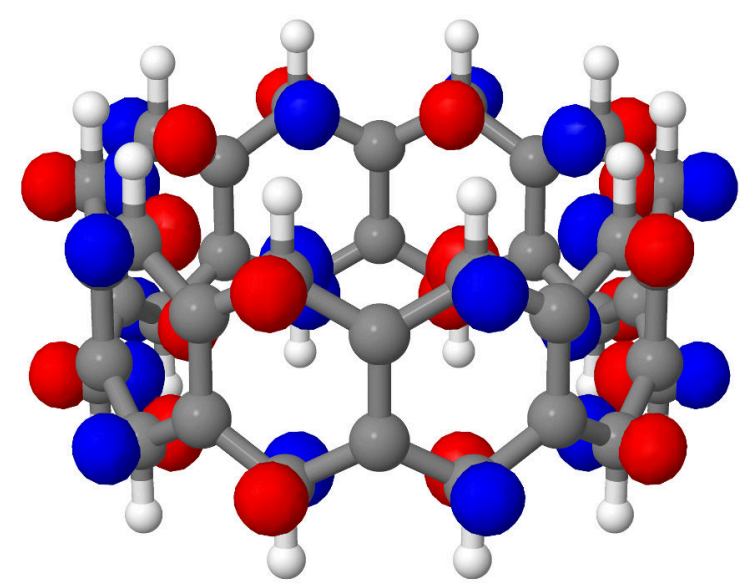

(a)

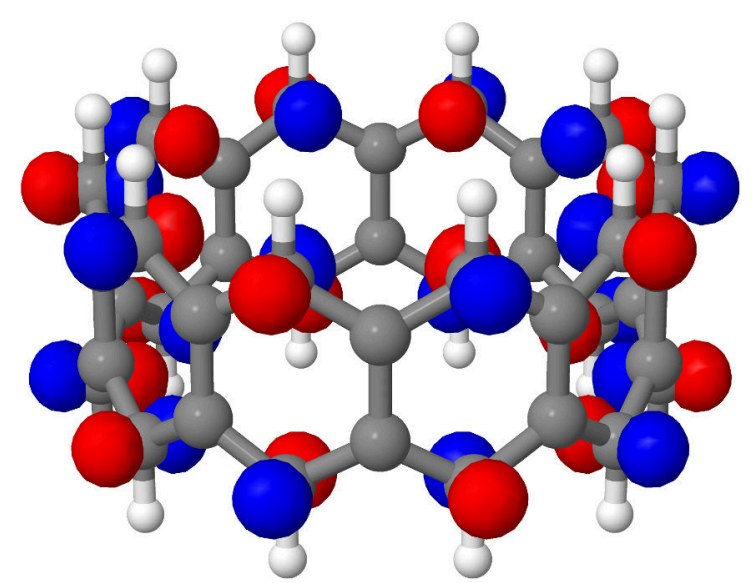

(b)

Figure 1: Symmetric (a) and antisymmetric (b) molecular orbitals constituting the HOMOLUMO pair for [10]cyclacene. Images created using the Jmol computer program, ${ }^{51}$ with a cutoff value of 0.04 . 


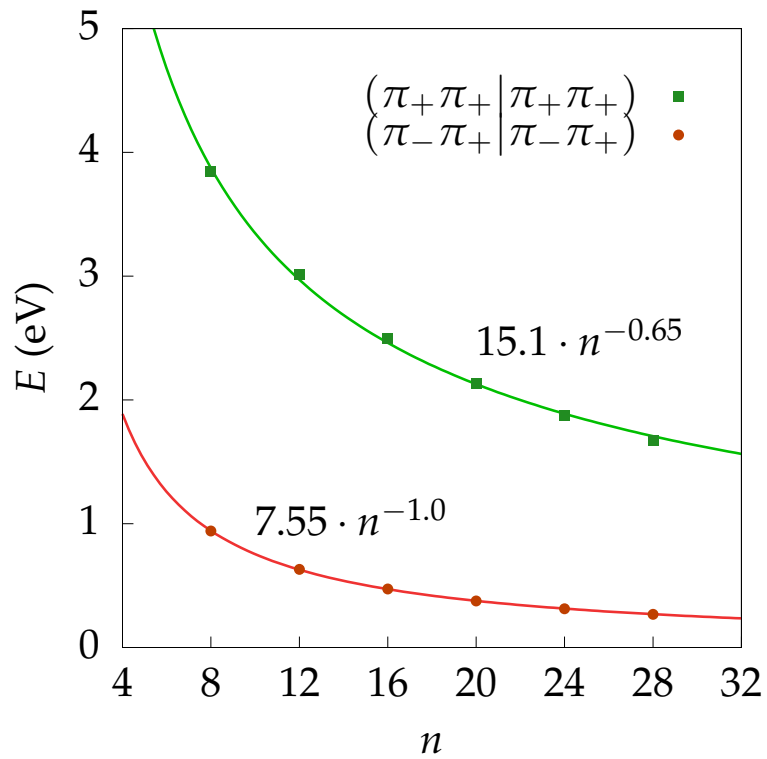

Figure 2: Values of two-electron integrals over $\operatorname{CASSCF}(2,2)$ natural orbitals plotted against the system size $n$. The green squares represent integrals over the symmetric orbital (corresponding to the Coulomb integral in Hartree-Fock nomenclature), while the red circles represent integrals over the symmetric and the antisymmetric orbitals (corresponding to the exchange integral in Hartree-Fock nomenclature). The calculations were run using the ANO-DZ basis set.

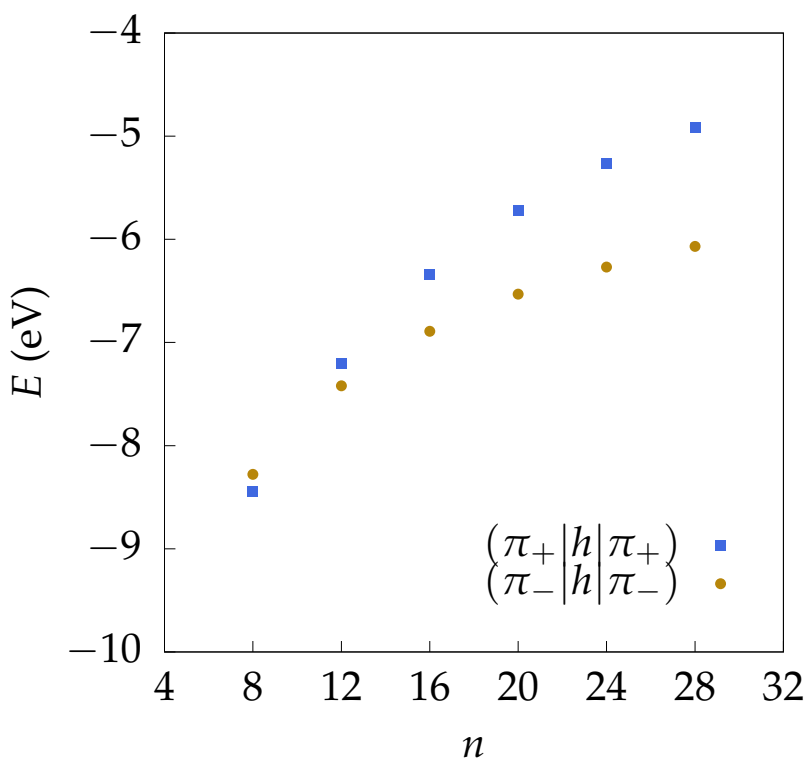

Figure 3: Values of one-electron integrals over $\operatorname{CASSCF}(2,2)$ natural orbitals plotted against the system size $n$. The blue squares represent integrals over the symmetric orbital, while the brown circles represent integrals over the antisymmetric orbitals. The calculations were run using the ANO-DZ basis set. 


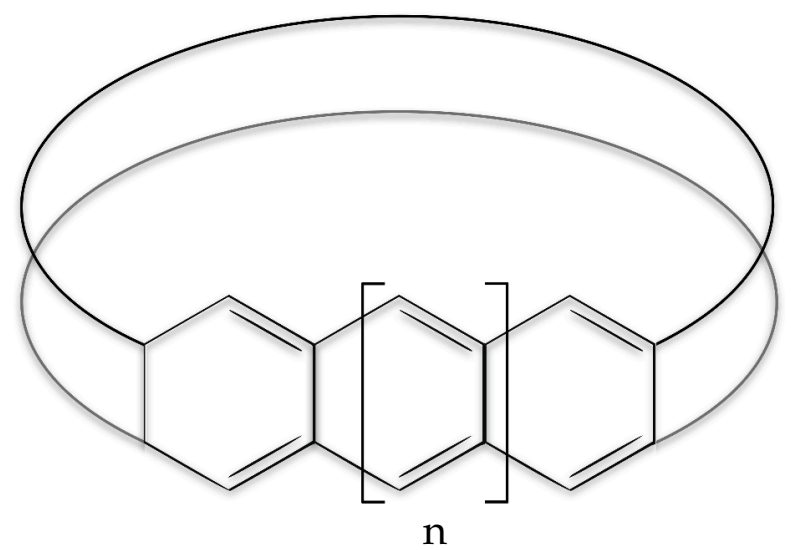

Figure 4: Schematic drawing of an $[n]$ cyclacene. The elementary unit is composed by four carbon atoms and is highlighted by the square brackets.
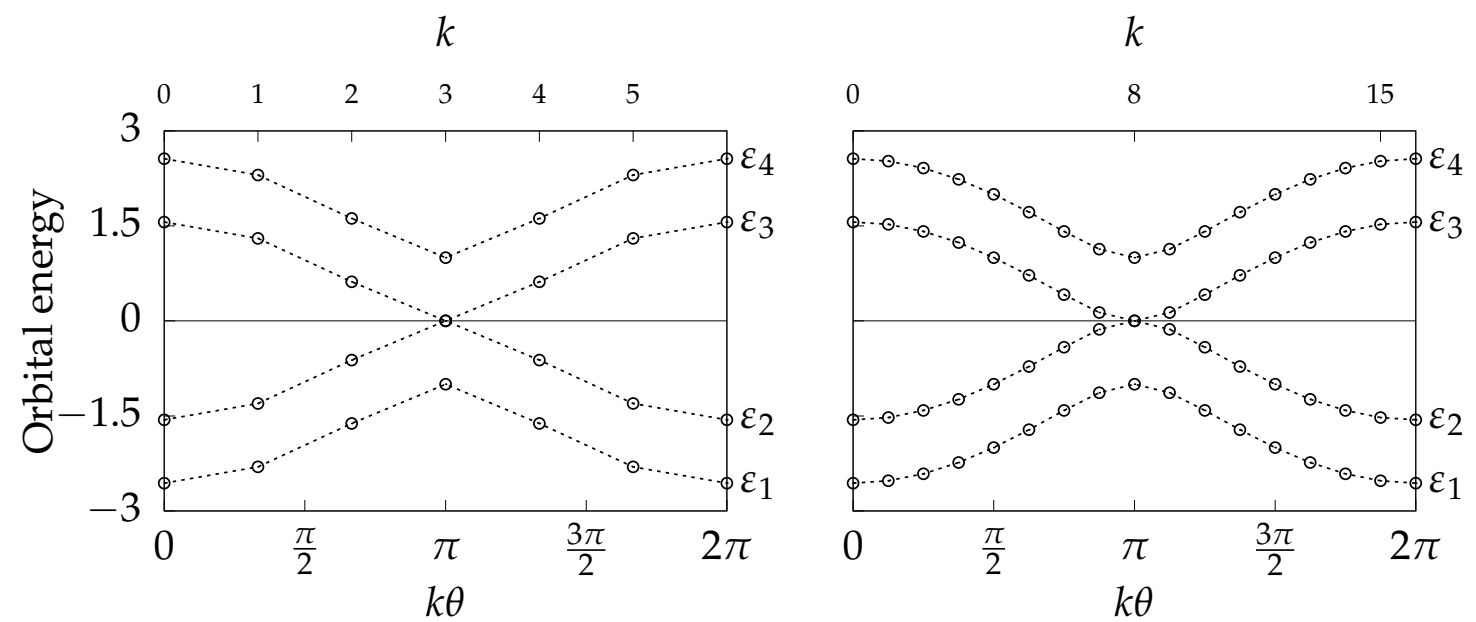

Figure 5: Hückel energy bands in arbitrary units for a [6]cyclacene (left) and for a [16]cyclacene (right) obtained by setting $\alpha=0$ and $\beta=1$. Note that the points at $k \theta=2 \pi$ are periodically repeated $\varepsilon_{j}$ which are the same as $\varepsilon_{j}(0)$. 


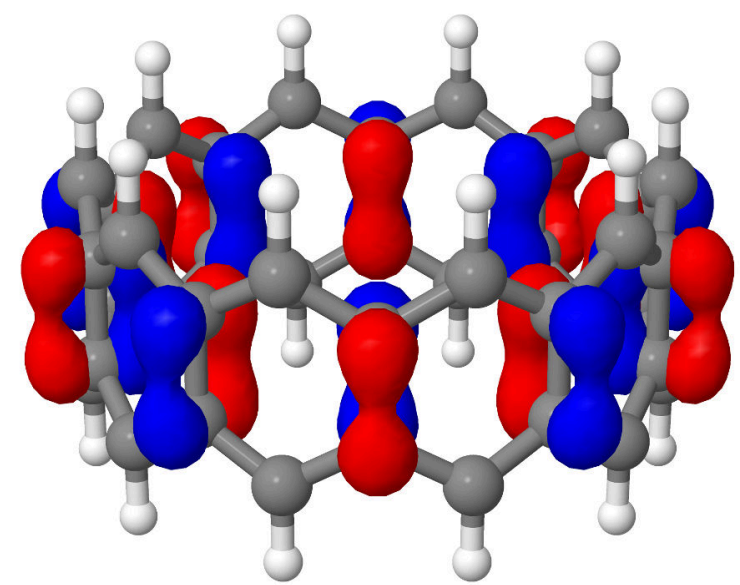

(a)

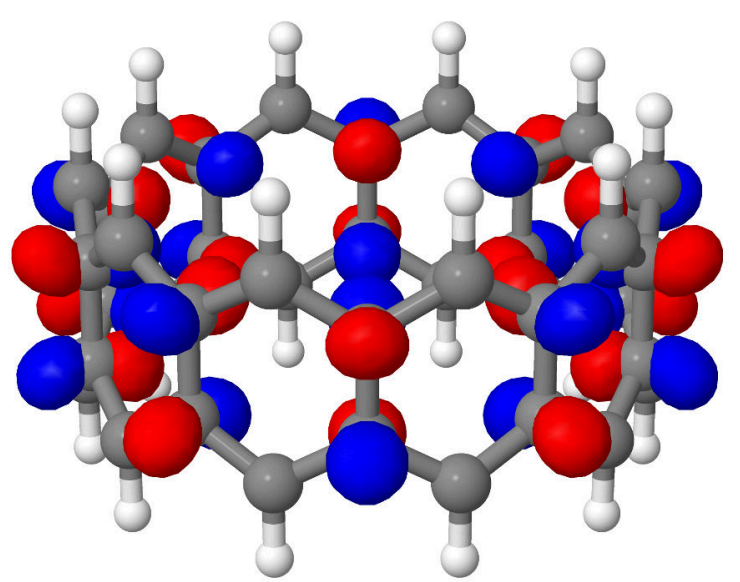

(b)

Figure 6: Molecular orbitals on the external energy bands for a [10]cyclacene. Figure (a) shows $\pi_{+}^{\prime}$ and Figure (b) shows $\pi_{-}^{\prime}$. Images created using the Jmol computer program, ${ }^{51}$ with a cutoff value of 0.04 .
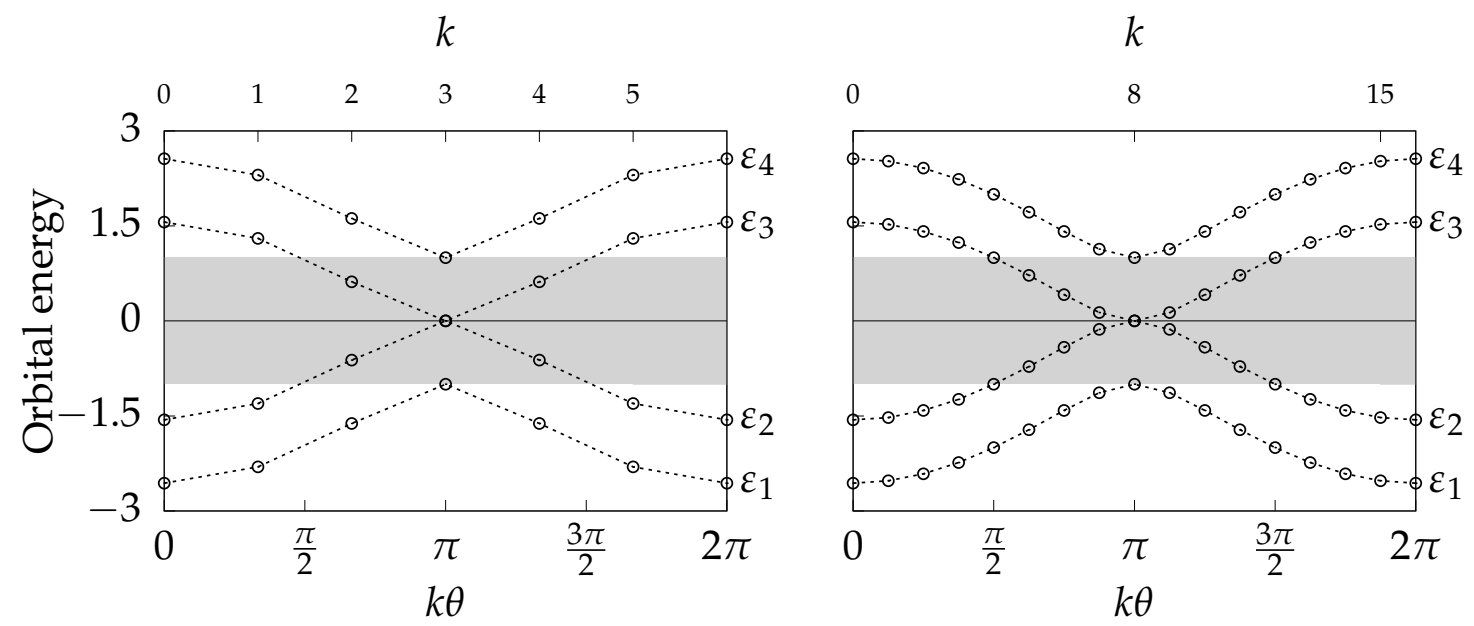

Figure 7: Hückel energy bands. The light gray zone delimits an energetic window containing a finite number of orbitals. Note that the points at $k \theta=2 \pi$ are periodically repeated $\varepsilon_{j}$ which are the same as $\varepsilon_{j}(0)$. 


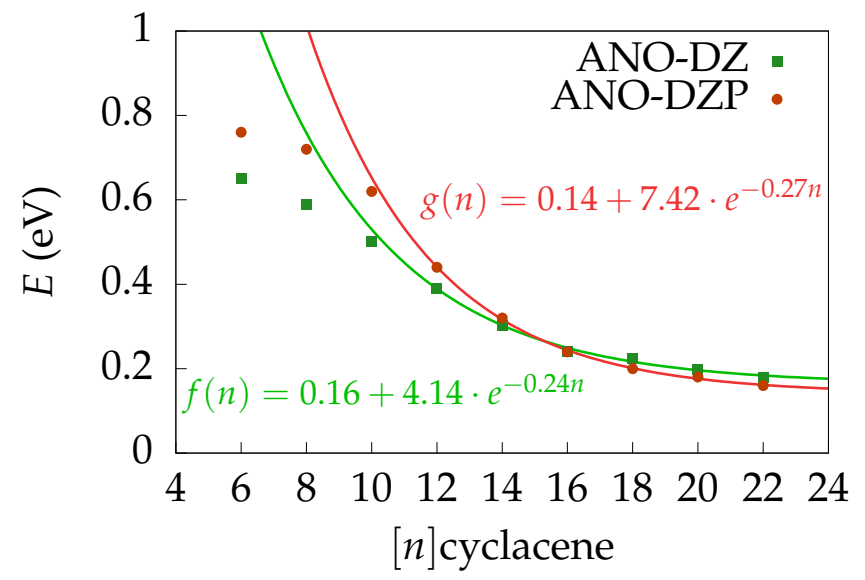

Figure 8: $\operatorname{CASSCF}(k, k)$ singlet-triplet energy gaps using the ANO-DZ (green squares) and the ANO-DZP (red circles) bases plotted against the system size $n$.

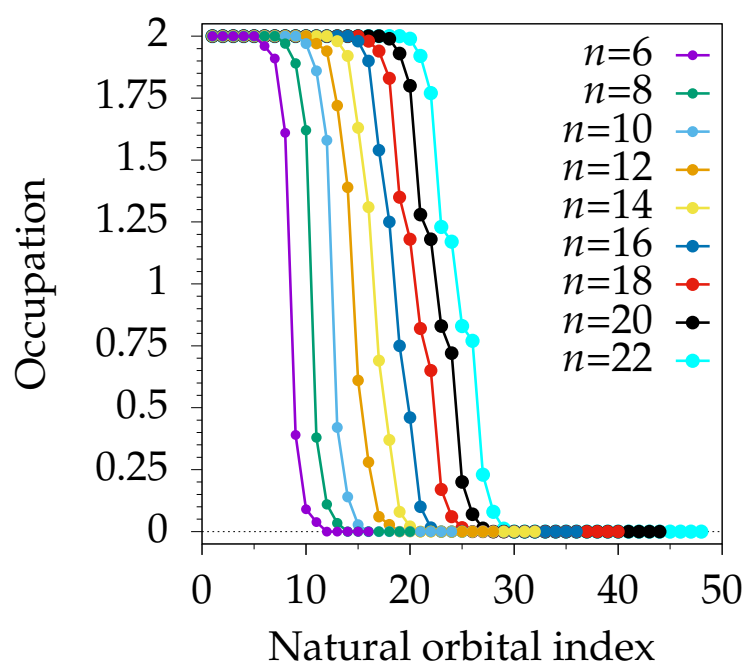

Figure 9: $\operatorname{CASSCF}(k, k)$ natural orbital occupation numbers using the ANO-DZP basis set. Note that all unique $\pi$ orbitals are plotted, including those not included in the active space. 


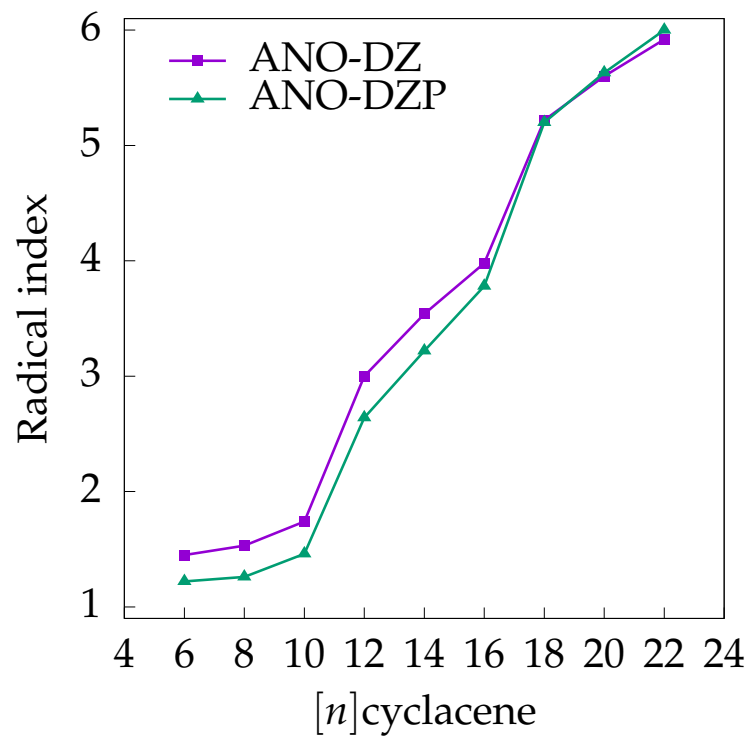

Figure 10: Effective number of unpaired electrons with respect to the system size. 


\section{References}

(1) Iijima, S. Helical Microtubules of Graphitic Carbon. Nature 1991, 354, 56-58.

(2) Heilbronner, E. Molecular Orbitals in Homologen Reihen Mehrkerniger Aromatischer Kohlenwasserstoffe: I. Die Eigenwerte von LCAO-MO's in Homologen Reihen. Helv. Chim. Acta 1954, 37, 921-935.

(3) Türker, L. Unusual Alternation of HMO Bond Orders in Cyclacenes. Polycycl. Aromat. Compd. 1996, 8, 67-71.

(4) Türker, L. AM1 Treatment of Hückel Type Cyclacenes. J. Mol. Struct. 1997, 407, 217220.

(5) Türker, L. MNDO Treatment of the Hückel and Möbius Types of Cyclacenes. J. Mol. Struct. THEOCHEM 1998, 454, 83-86.

(6) Türker, L. Zigzag Cyclopolyacenes: A Theoretical Study. J. Mol. Struct. THEOCHEM 1999, 491, 275-280.

(7) Gutman, I. Hückel Molecular Orbital Energies and Bond Orders of Cyclacenes. Polycycl. Aromat. Compd. 1996, 8, 251-257.

(8) Gutman, I.; Biedermann, P. U.; Ivanov-Petrović, V.; Agranat, I. Cyclic Conjugation Effects in Cyclacenes. Polycycl. Aromat. Compd. 1996, 8, 189-202.

(9) André, J.-M.; Champagne, B.; Perpète, E. A.; Guillaume, M. Linear, Cyclic, and Möbius Strip Polyacenes: The Influence of the Topology on the Size-Dependent HOMO-LUMO Energy Gap. Int. J. Quantum Chem. 2001, 84, 607-616.

(10) Guillaume, M.; Champagne, B.; Perpète, E. A.; André, J.-M. Möbius Strip Versus Linear and Cyclic Polyacenes: A Hückel and Semiempirical Investigation. Theor. Chem. Acc. 2001, 105, 431-436. 
(11) Choi, H. S.; Kim, K. S. Structures, Magnetic Properties, and Aromaticity of Cyclacenes. Angew. Chemie - Int. Ed. 1999, 38, 2256-2258.

(12) Houk, K. N.; Lee, P. S.; Nendel, M. Polyacene and Cyclacene Geometries and Electronic Structures: Bond Equalization, Vanishing Band Gaps, and Triplet Ground States Contrast with Polyacetylene. J. Org. Chem. 2001, 66, 5517-5521.

(13) Chen, Z.; Jiang, D. E.; Lu, X.; Bettinger, H. F.; Dai, S.; Schleyer, P. v. R.; Houk, K. N. Open-Shell Singlet Character of Cyclacenes and Short Zigzag Nanotubes. Org. Lett. $2007,9,5449-5452$.

(14) Sadowsky, D.; McNeill, K.; Cramer, C. J. Electronic Structures of [ $n]$-Cyclacenes ( $n=6-$ 12) and Short, Hydrogen-Capped, Carbon Nanotubules. Faraday Discuss. 2010, 145, 507-521.

(15) Wu, C.-S.; Lee, P.-Y.; Chai, J.-D. Electronic Properties of Cyclacenes from TAO-DFT. Nat. Sci. Reports 2016, 6, 37249.

(16) Hachmann, J.; Dorando, J. J.; Avilés, M.; Chan, G. K.-L. The Radical Character of the Acenes: A Density Matrix Renormalization Group Study. J. Chem. Phys. 2007, 127, 134309.

(17) Yang, Y.; Davidson, E. R.; Yang, W. Nature of Ground and Electronic Excited States of Higher Acenes. Proc. Natl. Acad. Sci. 2016, 113, E5098-E5107.

(18) Gleiter, R.; Esser, B.; Kornmayer, S. C. Cyclacenes: Hoop-Shaped Systems Composed of Conjugated Rings. Acc. Chem. Res. 2009, 42, 1108-1116.

(19) Eisenberg, D.; Shenhar, R.; Rabinovitz, M. Synthetic Approaches to Aromatic Belts: Building Up Strain in Macrocyclic Polyarenes. Chem. Soc. Rev. 2010, 39, 2879-2890.

(20) Omachi, H.; Segawa, Y.; Itami, K. Synthesis of Cycloparaphenylenes and Related 
Carbon Nanorings: A Step Toward the Controlled Synthesis of Carbon Nanotubes. Acc. Chem. Res. 2012, 45, 1378-1389.

(21) Scott, L. T. Conjugated Belts and Nanorings with Radially Oriented p Orbitals. Angew. Chemie - Int. Ed. 2003, 42, 4133-4135.

(22) Esser, B.; Rominger, F.; Gleiter, R. Synthesis of [6.8 $]_{3}$ Cyclacene: Conjugated Belt and Model for an Unusual Type of Carbon Nanotube. J. Am. Chem. Soc. 2008, 130, 6716-6717.

(23) Hitosugi, S.; Yamasaki, T.; Isobe, H. Bottom-up Synthesis and Thread-in-Bead Structures of Finite (n,0)-Zigzag Single-Wall Carbon Nanotubes. J. Am. Chem. Soc. 2012, $134,12442-12445$.

(24) Matsui, K.; Fushimi, M.; Segawa, Y.; Itami, K. Synthesis, Structure, and Reactivity of a Cylinder-Shaped Cyclo[12]orthophenylene[6]ethynylene: Toward the Synthesis of Zigzag Carbon Nanobelts. Org. Lett. 2016, 18, 5352-5355.

(25) Kintzel, O.; Luger, P.; Weber, M.; Schlüter, A.-D. Ring-Chain Equilibrium between an [18]Cyclacene Derivative and a Ladder Oligomer. Eur. J. Org. Chem. 1998, 1998, 99-105.

(26) Werner, H.-J.; Knowles, P. J.; Knizia, G.; Manby, F. R.; Schütz, M. Molpro: A GeneralPurpose Quantum Chemistry Program Package. Wiley Interdiscip. Rev. Comput. Mol. Sci. 2012, 2, 242-253.

(27) Werner, H.-J.; Knowles, P. J.; Knizia, G.; Manby, F. R.; Schütz, M.; Celani, P.; Győrffy, W.; Kats, D.; Korona, T.; Lindh, R. et al. MOLPRO, Version 2015.1, a Package of Ab Initio Programs. 2015; http://www.molpro.net.

(28) Widmark, P.-O.; Malmqvist, P.-Å.; Roos, B. O. Density Matrix Averaged Atomic 
Natural Orbital (ANO) Basis Sets for Correlated Molecular Wave Functions. Theor. Chim. Acta 1990, 77, 291-306.

(29) Becke, A. D. Density-Functional Exchange-Energy Approximation with Correct Asymptotic Behavior. Phys. Rev. A 1988, 38, 3098-3100.

(30) Becke, A. D. Density-Functional Thermochemistry. III. The Role of Exact Exchange. J. Chem. Phys. 1993, 98, 5648.

(31) Lee, C.; Yang, W.; Parr, R. G. Development of the Colle-Salvetti Correlation-Energy Formula into a Functional of the Electron Density. Phys. Rev. B 1988, 37, 785-789.

(32) Werner, H.-J.; Knowles, P. J. A Second Order Multiconfiguration SCF Procedure with Optimum Convergence. J. Chem. Phys. 1985, 82, 5053-5063.

(33) Knowles, P. J.; Werner, H.-J. An Efficient Second-Order MC SCF Method for Long Configuration Expansions. Chem. Phys. Lett. 1985, 115, 259-267.

(34) Angeli, C.; Cimiraglia, R.; Evangelisti, S.; Leininger, T.; Malrieu, J.-P. Introduction of n-Electron Valence States for Multireference Perturbation Theory. J. Chem. Phys. 2001, $114,10252$.

(35) Angeli, C.; Cimiraglia, R.; Malrieu, J.-P. n-Electron Valence State Perturbation Theory: A Spinless Formulation and an Efficient Implementation of the Strongly Contracted and of the Partially Contracted Variants. J. Chem. Phys. 2002, 117, 9138-9153.

(36) Angeli, C.; Pastore, M.; Cimiraglia, R. New Perspectives in Multireference Perturbation Theory: The n-Electron Valence State Approach. Theor. Chem. Acc. 2007, 117, 743-754.

(37) Mizukami, W.; Kurashige, Y.; Yanai, T. More $\pi$ Electrons Make a Difference: Emergence of Many Radicals on Graphene Nanoribbons Studied by Ab Initio DMRG Theory. J. Chem. Theory Comput. 2013, 9, 401-407. 
(38) Schriber, J. B.; Evangelista, F. A. Communication: An Adaptive Configuration Interaction Approach for Strongly Correlated Electrons with Tunable Accuracy. J. Chem. Phys. 2016, 144, 161106.

(39) Andersson, K.; Malmqvist, P.-Å.; Roos, B. O.; Sadlej, A. J.; Wolinski, K. Second-Order Perturbation Theory with a CASSCF Reference Function. J. Phys. Chem. 1990, 94, 5483-5488.

(40) Andersson, K.; Malmqvist, P.-Å.; Roos, B. O. Second-Order Perturbation Theory with a Complete Active Space Self-Consistent Field Reference Function. J. Chem. Phys. 1992, $96,1218-1226$.

(41) Roos, B. O.; Andersson, K. Multiconfigurational Perturbation Theory with Level Shift - the $\mathrm{Cr}_{2}$ Potential Revisited. Chem. Phys. Lett. 1995, 245, 215-223.

(42) Forsberg, N.; Malmqvist, P.-Å. Multiconfiguration Perturbation Theory with Imaginary Level Shift. Chem. Phys. Lett. 1997, 274, 196-204.

(43) Ghigo, G.; Roos, B. O.; Malmqvist, P. Å. A Modified Definition of the Zeroth-Order Hamiltonian in Multiconfigurational Perturbation Theory (CASPT2). Chem. Phys. Lett. 2004, 396, 142-149.

(44) Chai, J.-D. Density Functional Theory with Fractional Orbital Occupations. J. Chem. Phys. 2012, 136, 154104.

(45) Wu, C. S.; Chai, J.-D. Electronic Properties of Zigzag Graphene Nanoribbons Studied by TAO-DFT. J. Chem. Theory Comput. 2015, 11, 2003-2011.

(46) Fosso-Tande, J.; Nascimento, D. R.; DePrince III, A. E. Accuracy of Two-Particle NRepresentability Conditions for Describing Different Spin States and the Single-Triplet Gap in the Linear Acene Series. Mol. Phys. 2016, 114, 423-430. 
(47) Fosso-Tande, J.; Nguyen, T.-S.; Gidofalvi, G.; DePrince III, A. E. Large-Scale Variational Two-Electron Reduced-Density-Matrix-Driven Complete Active Space Self-Consistent Field Methods. J. Chem. Theory Comput. 2016, 12, 2260-2271.

(48) Head-Gordon, M. Characterizing Unpaired Electrons from the One-Particle Density Matrix. Chem. Phys. Lett. 2003, 372, 508-511.

(49) Ibeji, C. U.; Ghosh, D. Singlet-Triplet Gaps in Polyacenes: a Delicate Balance Between Dynamic and Static Correlations Investigated by Spin-Flip Methods. Phys. Chem. Chem. Phys. 2015, 17, 9849-9856.

(50) Hajgató, B.; Szieberth, D.; Geerlings, P.; De Proft, F.; Deleuze, M. S. A Benchmark Theoretical Study of the Electronic Ground State and of the Singlet-Triplet Split of Benzene and Linear Acenes. J. Chem. Phys. 2009, 131, 224321.

(51) Jmol: An Open-Source Java Viewer for Chemical Structures in 3D. http://www. jmol. org/. 


\section{Graphical TOC Entry}

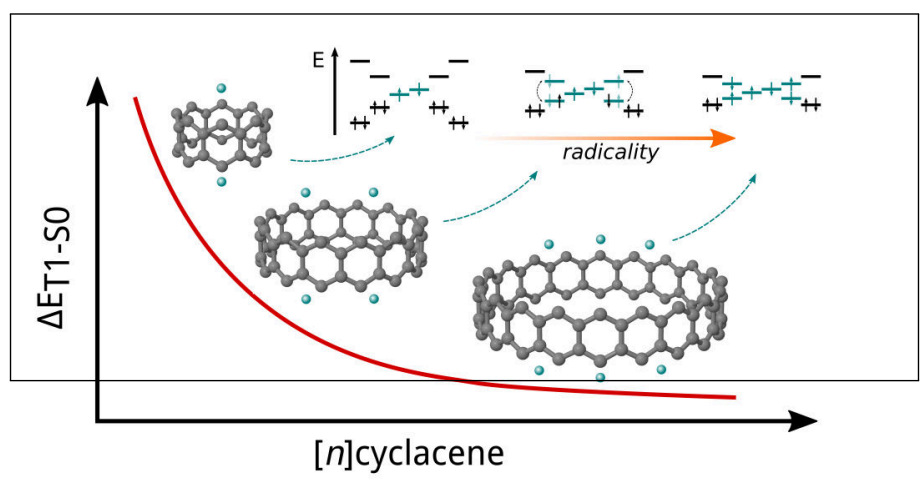

